PROCEEDINGS OF THE

AMERICAN MATHEMATICAL SOCIETY

Volume 133, Number 11, Pages 3263-3269

S 0002-9939(05)07860-3

Article electronically published on May 2, 2005

\title{
ON WANDERING VECTOR MULTIPLIERS FOR UNITARY GROUPS
}

\author{
GUOXING JI AND KICHI-SUKE SAITO
}

(Communicated by David R. Larson)

\begin{abstract}
A wandering vector multiplier is a unitary operator which maps the set of wandering vectors for a unitary system acting on a separable Hilbert space $\mathcal{H}$ into itself. It is proved that the wandering vector multipliers for a unitary group form a group, which gives a positive answer for a problem of Han and Larson. Furthermore, non-abelian unitary groups of order 6 are considered. We prove that the wandering vector multipliers of such a unitary group can not generate $\mathcal{B}(\mathcal{H})$. This negatively answers another of their problems.
\end{abstract}

\section{INTRODUCTION}

Let $\mathcal{H}$ be a separable Hilbert space. $\mathcal{B}(\mathcal{H})$ denotes the algebra of all bounded linear operators on $\mathcal{H}$. A unitary system $\mathcal{U}$ is a set of unitary operators acting on $\mathcal{H}$ which contains the identity $I$ in $\mathcal{B}(\mathcal{H})$.

A unit vector $\xi$ in $\mathcal{H}$ is called a wandering vector for $\mathcal{U}$ if $\mathcal{U} \xi=\{U \xi: U \in \mathcal{U}\}$ is an orthonormal set, that is, $(U \xi, V \xi)=0$ if $U, V \in \mathcal{U}$ and $U \neq V$. If $\mathcal{U} \xi$ is an orthonormal basis for $\mathcal{H}$, then $\xi$ is called a complete wandering vector for $\mathcal{U}$. The set of all complete wandering vectors for $\mathcal{U}$ is denoted by $\mathcal{W}(\mathcal{U})$. Let $A$ be a unitary operator on $\mathcal{H}$. We call $A$ a wandering vector multiplier for $\mathcal{U}$ if $A \mathcal{W}(\mathcal{U}) \subseteq \mathcal{W}(\mathcal{U})$ (1]). We use $\mathfrak{M}_{\mathcal{U}}$ to denote the set of all such operators. The wandering vectors and their multipliers for unitary systems have been systematically studied (see 1, 2, 3, 4]). One important class of unitary systems is that of unitary groups. Han and Larson in [3] considered the wandering vector multipliers for unitary groups and proved that for many unitary groups, for example, finite, abelian and ICC unitary groups, the wandering vector multipliers are groups. They asked in [3]:

(1) Do the wandering vector multipliers $\mathfrak{M}_{\mathcal{U}}$ for any unitary group $\mathcal{U}$ constitute a group?

If the unitary group is either abelian or ICC, then the set of wandering vector multipliers $\mathfrak{M}_{\mathcal{U}}$ generates $\mathcal{B}(\mathcal{H})([3])$. Thus they also asked:

(2) Does $\mathfrak{M}_{\mathcal{U}}$ generate $\mathcal{B}(\mathcal{H})$ in general?

Received by the editors April 30, 2003 and, in revised form, June 10, 2004.

2000 Mathematics Subject Classification. Primary 46L10, 46L51.

Key words and phrases. Unitary group, wandering vector, wandering vector multiplier, von Neumann algebra.

The first author was supported in part by the National Natural Science Foundation of China (No.10071047), the Excellent Young Teachers Program of MOE, P.R.C and China Scholarship Council. The second author was supported in part by Grants-in-Aid for Scientific Research, Japan Society for the Promotion of Science. 
In this note we consider these two problems and give a positive answer for the first problem, a negative answer for the second problem.

Let $\mathcal{S}$ be a subset of $\mathcal{B}(\mathcal{H})$. We use $W^{*}(\mathcal{S})$ to denote the von Neumann algebra generated by $\mathcal{S}$. The commutant of $\mathcal{S}$ is $\mathcal{S}^{\prime}=\{T \in \mathcal{B}(\mathcal{H}): T A=A T, \forall A \in \mathcal{S}\}$. Let $x \in \mathcal{H}$ be a nonzero vector. The local commutant $\mathcal{C}_{x}(\mathcal{S})$ of $\mathcal{S}$ at $x$ is defined by $\{T \in \mathcal{B}(\mathcal{H}): T A x=A T x, \forall A \in \mathcal{S}\}$. A very useful result is the one-to-one correspondence between the complete wandering vectors and the unitary operators in $\mathcal{C}_{\xi}(\mathcal{U})$. In particular, if $\xi \in \mathcal{W}(\mathcal{U})$, then $\mathcal{W}(\mathcal{U})=\left\{T \xi: T \in \mathcal{C}_{\xi}(\mathcal{U})\right.$ is unitary $\}$ (see [1, Proposition 1.3]).

Let $\mathcal{M}$ be a finite von Neumann algebra on $\mathcal{H}$ and $\tau$ a faithful normal finite trace on $\mathcal{M}$. For $1 \leq p \leq \infty$, let $\mathbb{L}^{p}(\mathcal{M})$ denote the non-commutative $L^{p}$-space associated with $\mathcal{M}$ and $\tau$ (see $\left[\underline{8}\right.$ ). We use $\|\cdot\|_{p}$ to denote the norm of $\mathbb{L}^{p}(\mathcal{M})$. Then $\mathbb{L}^{2}(\mathcal{M})$ is a Hilbert space with the inner product $(x, y)=\tau\left(y^{*} x\right)$, for all $x, y \in \mathbb{L}^{2}(\mathcal{M})$. Moreover, for any $A \in \mathcal{M}$, we define the operators $L_{A}$ and $R_{A}$ on $\mathbb{L}^{2}(\mathcal{M})$ by the equations

$$
L_{A} x=A x, \quad R_{A} x=x A, \quad \forall x \in \mathbb{L}^{2}(\mathcal{M}) .
$$

Then we know that $\mathcal{M}$ is $*$-isomorphic (resp. $*$-anti-isomorphic ) to $L(\mathcal{M})=\left\{L_{A}\right.$ : $A \in \mathcal{M}\}\left(\operatorname{resp} . R(\mathcal{M})=\left\{R_{A}: A \in \mathcal{M}\right\}\right)$ and $(L(\mathcal{M}))^{\prime}=R(\mathcal{M})$. In particular, if $\mathcal{M}$ has a cyclic and separate unit vector $\xi$ and we define $\tau(A)=(A \xi, \xi)$, for all $A \in \mathcal{M}$, then $\mathcal{H}$ is isomorphic to $\mathbb{L}^{2}(\mathcal{M})$ and $\mathcal{M}$ is unitarily equivalent to $L(\mathcal{M})$ (see [8, Theorem 7.2.9]). In this case, we may assume that $\mathcal{H}=\mathbb{L}^{2}(\mathcal{M})$ and $\mathcal{M}$ identifies with $L(\mathcal{M})$.

\section{AN ANSWER FOR THE FIRST PROBLEM}

Let $\mathcal{U}_{1}$ and $\mathcal{U}_{2}$ acting on Hilbert spaces $\mathcal{H}_{1}$ and $\mathcal{H}_{2}$, respectively, be two unitary systems. We say that $\mathcal{U}_{1}$ and $\mathcal{U}_{2}$ are unitarily equivalent if there is a unitary operator $T: \mathcal{H}_{1} \rightarrow \mathcal{H}_{2}$ such that $T \mathcal{U}_{1} T^{*}=\mathcal{U}_{2}$. Then we easily have

Proposition 1 (See [3]). Let $\mathcal{U}_{1}$ and $\mathcal{U}_{2}$ be two unitarily equivalent unitary systems on Hilbert spaces $\mathcal{H}_{1}$ and $\mathcal{H}_{2}$ respectively. If one has a completely wandering vector, so does the other and their wandering vector multipliers $M_{\mathcal{U}_{1}}$ and $M_{\mathcal{U}_{2}}$ are unitarily equivalent.

Let $\mathcal{U}$ be a unitary group on $\mathcal{H}$ with a completely wandering vector $\xi$ and put $\mathcal{M}=W^{*}(\mathcal{U})$. Then by Proposition 1.1 in $[3$, we have that $\mathcal{M}$ is finite with the cyclic and separate vector $\xi$. Letting $\xi, \eta \in \mathcal{W}(\mathcal{U})$, we have $\tau(T)=(T \xi, \xi)=(T \eta, \eta)$ for all $T \in \mathcal{M}$ and $\tau$ is a faithful normal finite trace of $\mathcal{M}$. Thus we may assume that $\mathcal{H}=\mathbb{L}^{2}(\mathcal{M})$ and $\mathcal{M}$ identifies with $L(\mathcal{M})$ by Proposition 1. Now we give a positive answer for the first problem, which is posed by Han and Larson in [3].

Theorem 1. Let $\mathcal{U}$ be a unitary group with a completely wandering vector $\xi$. Then $\mathfrak{M}_{\mathcal{U}}$ is a group.

Proof. By Proposition 1, without loss of generality, we may asssume $\mathcal{H}=\mathbb{L}^{2}(\mathcal{M})$ and $I \in \mathbb{L}^{2}(\mathcal{M})$ is a completely wandering vector for $\mathcal{U}$, that is, $I \in \mathcal{W}(\mathcal{U})$.

Let $\Phi \in \mathfrak{M}_{\mathcal{U}}$. It suffices to show that $\Phi^{-1}$ is also a wandering vector multiplier. $\forall x \in \mathcal{W}(\mathcal{U})$, by Lemma 1.1 in [1], there is a unitary operator $T \in \mathcal{U}^{\prime}$ such that $x=T I$. We note that $\mathcal{U}^{\prime}=\mathcal{M}^{\prime}=R(\mathcal{M})$. Then there is a unitary operator $U \in \mathcal{M}$ such that $x=R_{U} I=I U=U$, that is, $\mathcal{W}(\mathcal{U})=\{x: x$ is a unitary operator in $\mathcal{M}\}$ (see Lemma 2.2 in [3]). Thus $\Phi$ is a unitary-preserving linear map on $\mathcal{M}$. It 
follows that $\Phi(I)$ is unitary in $\mathcal{M}$. Replacing $\Phi$ by $(\Phi(I))^{-1} \Phi$, we can assume that $\Phi(I)=I$. By Proposition 2.3, Proposition 2.5 and Corollary 3.6 in [3], we know that $\Phi$ is an isometry as well as a Jordan homomorphism from $\mathcal{M}$ into $\mathcal{M}$. Let $\mathcal{B}$ be the von Neumann algebra generated by $\Phi(\mathcal{M})$. Then we have that $\mathcal{B}=\mathcal{M}$ by the fact that $\Phi(\mathcal{M})$ is dense in $\mathbb{L}^{2}(\mathcal{M})$ (see the first part of the proof of Theorem 2.9 in [3]). By Lemma 1.4 in [3], we know that there exists a projection $P$ in the center $\mathcal{Z}(\mathcal{M})$ of $\mathcal{M}$, such that

$$
a \rightarrow \Phi(a) P
$$

is a $*$-homomorphism, and

$$
a \rightarrow \Phi(a)(I-P)
$$

is a $*$-antihomomorphism.

Let $\Phi_{1}(a)=\Phi(a) P$ and $\Phi_{2}(a)=\Phi(a)(I-P)$ for all $a \in \mathcal{M}$ and denote by $\operatorname{ker}\left(\Phi_{i}\right)$ the kernel of $\Phi_{i}$ for $i=1,2$. We claim that $\operatorname{ker}\left(\Phi_{i}\right)(i=1,2)$ is weakly closed.

Let $x_{\lambda}$ be a net in $\operatorname{ker}\left(\Phi_{1}\right)$ such that $\lim _{\lambda} x_{\lambda}=x$ in the weak operator topology for some $x \in \mathcal{M}$. We next show that $x \in \operatorname{ker}\left(\Phi_{1}\right)$. For any $y \in \mathbb{L}^{2}(\mathcal{M})$, we have

$$
\begin{aligned}
& \lim _{\lambda}\left(\Phi\left(x_{\lambda}\right), y\right)=\lim _{\lambda}\left(x_{\lambda}, \Phi^{*}(y)\right) \\
= & \lim _{\lambda}\left(x_{\lambda} I, \Phi^{*}(y)\right)=\left(x, \Phi^{*}(y)\right)=(\Phi(x), y),
\end{aligned}
$$

which implies that $\Phi\left(x_{\lambda}\right) \rightarrow \Phi(x)$ weakly in $\mathbb{L}^{2}(\mathcal{M})$. We then have $P \Phi\left(x_{\lambda}\right) \rightarrow$ $P \Phi(x)$ weakly. Therefore $P \Phi(x)=0$ since $P \Phi\left(x_{\lambda}\right)=0$. Thus $x \in \operatorname{ker}\left(\Phi_{1}\right)$, that is, $\operatorname{ker}\left(\Phi_{1}\right)$ is weakly closed. Similarly we also have that $\operatorname{ker}\left(\Phi_{2}\right)$ is weakly closed. Since $\Phi_{1}$ (resp. $\Phi_{2}$ ) is a $*$-homomorphism (resp. *-antihomomorphism), $\operatorname{ker} \Phi_{1}$ (resp. ker $\Phi_{2}$ ) is a weakly closed two-sided ideal of $\mathcal{M}$. By Theorem 6.8.8 in [7], there are two central projections $E, F \in \mathcal{Z}(\mathcal{M})$ such that $\operatorname{ker}\left(\Phi_{1}\right)=E \mathcal{M}$ and $\operatorname{ker}\left(\Phi_{2}\right)=F \mathcal{M}$. Since $\Phi=\Phi_{1}+\Phi_{2}$ and $\Phi(I)=I$, We have $\Phi_{1}(I-E)=P$ and $\Phi_{2}(I-F)=I-P$. We easily have $E F=0$ since $\Phi(E F)=\Phi_{1}(E F)+\Phi_{2}(E F)=0$. Thus $E$ and $F$ are orthogonal to each another.

Next we prove that $E+F=I$. We first claim that $\Phi_{1}$ (resp. $\Phi_{2}$ ) may be extended to a contraction on $\mathbb{L}^{2}(\mathcal{M})$. In fact, we have $\Phi_{1}(x)=\Phi(x) P$ for all $x \in \mathcal{M}$. Then for any $x \in \mathbb{L}^{2}(\mathcal{M})$, there is a net $x_{\lambda}$ in $\mathcal{M}$ such that $\lim _{\lambda} x_{\lambda}=x$ in $\mathbb{L}^{2}(\mathcal{M})$. It follows that $\lim _{\lambda} \Phi\left(x_{\lambda}\right)=\Phi(x)$ in $\mathbb{L}^{2}(\mathcal{M})$. Thus $\lim _{\lambda} \Phi_{1}\left(x_{\lambda}\right)=\lim _{\lambda} \Phi\left(x_{\lambda}\right) P=\Phi(x) P$ in $\mathbb{L}^{2}(\mathcal{M})$. We have that $\Phi_{1}$ may be extended to a bounded linear operator on $\mathbb{L}^{2}(\mathcal{M})$ and we also denote it by $\Phi_{1}$. It is clear that $\left\|\Phi_{1}\right\| \leq\|\Phi\| \leq 1$. We similarly have that $\Phi_{2}$ may be extended to a contraction on $\mathbb{L}^{2}(\mathcal{M})$ and we also denote it by $\Phi_{2}$. Then we have that $\Phi=\Phi_{1}+\Phi_{2}$ on $\mathbb{L}^{2}(\mathcal{M})$.

Note that $\Phi$ is a unitary operator on $\mathbb{L}^{2}(\mathcal{M})$ and $\mathcal{M} \subseteq \mathbb{L}^{2}(\mathcal{M})$. Then there is a vector $x \in \mathbb{L}^{2}(\mathcal{M})$ such that $\Phi(x)=P$. Now, $\Phi(x)=\Phi(x) P+\Phi(x)(I-P)=$ $\Phi_{1}(x)+\Phi_{2}(x)=P$, so we have $\Phi_{2}(x)=0$. Then $x \in \operatorname{ker}\left(\Phi_{2}\right)=F \mathbb{L}^{2}(\mathcal{M})$ in $\mathbb{L}^{2}(\mathcal{M})$. Then $x=F x=x F$. On the other hand, $\Phi(x)=P=\Phi_{1}(I-E)=\Phi_{1}(x)$; then $I-E-x \in \operatorname{ker}\left(\Phi_{1}\right)=\mathbb{L}^{2}(\mathcal{M}) E$. It now follows that $(I-E-x)(I-E)=0$, that is, $x(I-E)=I-E$. Thus we have $x=x E+(I-E)=x F$. Hence $x=F$ since $E F=0$. Now we have $\Phi(F)=\Phi_{1}(F)=\Phi_{1}((I-E))$ and $F \leqq I-E$. It follows that $F=I-E \operatorname{since} \operatorname{ker}\left(\Phi_{1}\right)=E \mathcal{M}$. We thus have $E+F=I$. It also follows that $\Phi(\mathcal{M})=\Phi_{1}(\mathcal{M})+\Phi_{2}(\mathcal{M})$. 
It now follows that $\Phi_{1}$ is an injective $*$ - homomorphism from $F \mathcal{M}$ into $P \mathcal{M}$. Thus $\Phi_{1}(F \mathcal{M})$ is a $*$-subalgebra of $P \mathcal{M}$. We claim that $\Phi_{1}(F \mathcal{M})=P \mathcal{M}$. By the proof above we know that the von Neumann algebra generated by $\Phi(\mathcal{M})$ is $\mathcal{M}$. Then $\Phi_{1}(F \mathcal{M})$ must be strongly dense in $P \mathcal{M}$ since $\Phi(\mathcal{M})=\Phi_{1}(\mathcal{M})+\Phi_{2}(\mathcal{M}) \subset$ $P \mathcal{M}+(I-P) \mathcal{M}=\mathcal{M}$. Let $B \in P \mathcal{M}$ with norm one. Then there is a net $\left\{A_{\lambda}\right\}$ in $F \mathcal{M}$ such that $\Phi_{1}\left(A_{\lambda}\right)$ is in the unit ball of $\Phi_{1}(F \mathcal{M})$ and $\Phi_{1}\left(A_{\lambda}\right)$ converges to $B$ in the strong operator topology by Kaplansky's density theorem. Thus $\left\{A_{\lambda}\right\}$ is contained in the unit ball of $F \mathcal{M}$ because $\Phi$ is an isometry for the norm topology of $\mathcal{M}$. Note that $\left\|\Phi_{1}\left(A_{\lambda}\right)-B\right\|_{2}=\left\|\Phi\left(A_{\lambda}\right) P-B P\right\|_{2} \rightarrow 0$ in $\mathbb{L}^{2}(\mathcal{M})$. We have that $\Phi_{1}\left(A_{\lambda}\right)$ is a Cauchy net in $\mathbb{L}^{2}(\mathcal{M})$. However we have $\Phi_{1}(X)=\Phi(X)$ for all $X \in F \mathcal{M}$ and $\Phi$ is a unitary operator on $\mathbb{L}^{2}(\mathcal{M})$. Then $A_{\lambda}$ is also a Cauchy net in $\mathbb{L}^{2}(\mathcal{M})$. Letting $A_{\lambda} \rightarrow A$ in $\mathbb{L}^{2}(\mathcal{M})$, we have $\Phi_{1}\left(A_{\lambda}\right)=\Phi\left(A_{\lambda}\right) \rightarrow \Phi(A)$ in $\mathbb{L}^{2}(\mathcal{M})$. It follows that $B=\Phi(A)=\Phi_{1}(A)$. On the other hand, since the net $\left\{A_{\lambda}\right\}$ is in the unit ball of $F \mathcal{M}$, we may assume that $A_{\lambda}$ converges to an operator $A^{\prime} \in F \mathcal{M}$ in the weak operator topology. Then we easily have $A=A^{\prime} \in F \mathcal{M}$ and thus $\Phi_{1}(F \mathcal{M})=P \mathcal{M}$.

Similarly we have $\Phi_{2}((I-F) \mathcal{M})=(I-P) \mathcal{M}$. Then $\Phi$ is a surjective isometry on $\mathcal{M}$. So the inverse of $\Phi$ is also an isometry. By Lemma 12 in $\underline{6}, \Phi^{-1}$ preserves unitary elements. Then $\Phi^{-1}$ is a wandering vector multiplier, that is, $\Phi^{-1} \in \mathfrak{M}_{\mathcal{U}}$. The proof is complete.

Corollary 1. Let $\Phi \in \mathfrak{M}_{\mathcal{U}}$. Then $\Phi$ is a weakly continuous and surjective isometry on $\mathcal{M}$. Moreover, if $\Phi(I)=I$, then $\tau(\Phi(T))=\tau(T)$ for all $T \in \mathcal{M}$.

Proof. By the proof of Theorem 1, we know that $\Phi_{1}$ (resp. $\left.\Phi_{2}\right)$ is a $*$-isomorphism (resp. *-anti-isomorphism) from the von Neumann algebra $F \mathcal{M}$ (resp. $(I-F) \mathcal{M})$ onto $P \mathcal{M}$ (resp. $(I-P) \mathcal{M}$ ). Then both $\Phi_{1}$ and $\Phi_{2}$ are ultraweakly continuous. Hence $\Phi=\Phi_{1}+\Phi_{2}$ is also ultraweakly continuous. Ultraweak topology coincides with weak topology on $\mathcal{M}$ since $\mathcal{M}$ has a cyclic and separate vector (see [7]); then $\Phi$ is weakly continuous. We now show that $\tau(\Phi(T))=\tau(T)$ for all $T \in \mathcal{M}$ if $\Phi$ is a Jordan homomorphism such that $\Phi(I)=I$. In fact, since $\tau(\cdot)$ is a trace and $P$ is a central projection, we have

$$
\begin{aligned}
& \tau\left(\Phi\left(T^{*} T\right)\right)=\tau\left(\Phi\left(T^{*} T\right) P\right)+\tau\left(\Phi\left(T^{*} T\right)(I-P)\right) \\
= & \tau\left(\Phi\left(T^{*}\right) \Phi(T) P\right)+\tau\left(\Phi(T) \Phi\left(T^{*}\right)(I-P)\right) \\
= & \tau\left(\Phi(T)^{*} \Phi(T) P\right)+\tau\left(\Phi(T) \Phi(T)^{*}(I-P)\right) \\
= & \tau\left(\Phi\left(T^{*}\right) \Phi(T) P\right)+\tau\left(\Phi(T)^{*} \Phi(T)(I-P)\right) \\
= & \tau\left(\Phi\left(T^{*}\right) \Phi(T)\right) \\
= & \|\Phi(T)\|_{2}^{2}=\|T\|_{2}^{2} \\
= & \tau\left(T^{*} T\right) .
\end{aligned}
$$

It follows that $\tau(\Phi(T))=\tau(T)$ for all positive elements $T \in \mathcal{M}$ and therefore for all elements $T \in \mathcal{M}$. The proof is complete.

Let $\mathcal{J}_{\mathcal{U}}$ be the set of all weakly continuous and surjective Jordan homomorphisms $\Phi$ on $\mathcal{M}$ such that $\tau \circ \Phi=\tau$.

Theorem 2. $\mathfrak{M}_{\mathcal{U}}$ is unitarily equivalent to the group generated by $\mathcal{J}_{\mathcal{U}}$ and all the unitary elements in $\mathcal{M}$. 
Proof. By Proposition 1, Theorem 1 and Corollary 1, it is sufficient to show that $\mathcal{J}_{\mathcal{U}} \subset \mathfrak{M}_{\mathcal{U}}$. Let $\Phi \in \mathcal{J}_{\mathcal{U}}$. Then it is clear that $\Phi$ preserves the unitary elements of $\mathcal{M}$. So it is enough to prove that $\Phi$ can be extended to a unitary operator on $\mathbb{L}^{2}(\mathcal{M})$. Letting $X \in \mathcal{M}$, as in the proof of Corollary 1, we have

$$
\|\Phi(X)\|_{2}^{2}=\tau\left(\Phi(X)^{*} \Phi(X)\right)=\tau\left(\Phi\left(X^{*} X\right)\right)=\tau\left(X^{*} X\right)=\|X\|_{2}^{2} .
$$

Then $\Phi$ can be extended to a unitary operator on $\mathbb{L}^{2}(\mathcal{M})$ and $\Phi \in \mathfrak{M}_{\mathcal{U}}$. The proof is complete.

Let $U, V \in \mathcal{B}(\mathcal{H})$ be two unitary operators. Let $\theta \in(0,1)$ and let $\lambda=\exp (2 \pi i \theta)$. If $U V=\lambda V U$, then we call the unitary system $\mathcal{U}_{U, V}=\left\{U^{m} V^{n}: m, n \in \mathbb{Z}\right\}$ a rotation system. It is not a unitary group. If $\theta$ is an irrational number, it is called an irrational rotation system, which has been studied systematically in [2, 3]. It is proved that the wandering vector multipliers of $\mathcal{U}_{U, V}$ form a group since $W^{*}\left(\mathcal{U}_{U, V}\right)$ is a factor (Theorem $4.1 \mathrm{in} \mathrm{[3]).} \mathrm{Contrasting} \mathrm{this} \mathrm{with} \mathrm{the} \mathrm{irrational} \mathrm{rotation} \mathrm{system,}$ if $\theta$ is a rational number in $(0,1)$, we note that the von Neumann algebra generated by $U$ and $V$ is not a factor in general. In [4, Theorem 4.3], Han and Larson proved that $\mathcal{W}\left(\mathcal{U}_{U, V}\right)$ is a closed and connected subset of $\mathcal{H}$. We similarly have the following result whose proof is similar to that of Theorem 1 above. We give the theorem here without proof.

Theorem 3. Let $\theta$ be a rational number in $(0,1)$. If $\mathcal{U}_{U, V}$ has a completely wandering vector, then $\mathfrak{M}_{\mathcal{U}_{U, V}}$ is a group.

\section{AN ANSWER FOR THE SECOND PROBLEM}

We give a counterexample in this section to show that the second problem is false in general. Let $G$ be a finite group. If $G$ is non-abelian, then it is at least of order 6 ([5, p. 98]). Furthermore, any two non-abelian groups of order 6 are isomorphic. Let $\mathcal{U}$ be a non-abelian unitary group of order 6 acting on the Hilbert space $\mathcal{H}$ such that $\mathcal{W}(\mathcal{U}) \neq \emptyset$. Then $\operatorname{dim} \mathcal{H}=6$. We first note that, up to unitary equivalence, such a unitary group is unique.

Proposition 2. Let $\mathcal{U}_{i}$ be two non-abelian unitary groups of order 6 acting on the Hilbert space $\mathcal{H}_{i}$ such that $\mathcal{W}\left(\mathcal{U}_{i}\right) \neq \emptyset$ for $i=1,2$. Then $W^{*}\left(\mathcal{U}_{1}\right)$ and $W^{*}\left(\mathcal{U}_{2}\right)$ are unitarily equivalent.

Proof. Let $\xi_{i}(i=1,2)$ be the completely wandering vector for $\mathcal{U}_{i}$. Then $\xi_{i}$ is cyclic and separate for $W^{*}\left(\mathcal{U}_{i}\right)$ by Proposition 1.1 in [3]. Noting that $\mathcal{U}_{1}$ and $\mathcal{U}_{2}$ are group isomorphic, then we easily have that $W^{*}\left(\mathcal{U}_{1}\right)$ and $W^{*}\left(\mathcal{U}_{2}\right)$ are $*$-isomorphic. Thus we have the result by Theorem 7.2.9 in [7]. The proof is complete.

By Proposition 2 above, it is sufficient to consider a concrete non-abelian unitary group of order 6 with a completely wandering vector. Let $\mathbb{C}^{n}$ be the complex $n$ dimensional Hilbert space, and we denote by $M_{n}$ the set of all complex $n \times n$ matrices. $I_{n} \in M_{n}$ is the identity matrix.

Let $\mathcal{H}=\mathbb{C}^{6}$ and let $\left\{e_{i}: i=1,2, \cdots, 6\right\}$ be an orthonormal basis of $\mathbb{C}^{6}$, and for $x, y \in \mathbb{C}^{6}$, define $x \otimes y(z)=(z, y) x$, for all $z \in \mathbb{C}^{6}$. Then $x \otimes x$ is a rank-1 projection for all unit vectors $x$.

Let $\lambda=\exp \left(\frac{2}{3} \pi i\right)=-\frac{1}{2}+i \frac{\sqrt{3}}{2}$ and let $A_{1}=\lambda e_{1} \otimes e_{1}+\bar{\lambda} e_{2} \otimes e_{2}$ and $A_{2}=$ $e_{1} \otimes e_{2}+e_{2} \otimes e_{1}$ be two unitary matrices in $M_{2}$. It is easy to check that $A_{1}^{3}=I_{2}$, 
$A_{1}^{2} \neq I_{2}, A_{2}^{2}=I_{2}$ and $A_{2} A_{1}=A_{1}^{-1} A_{2}$. Then by Theorem 6.13 in [5], we know that the group $G$ generated by $A_{1}$ and $A_{2}$ is non-abelian with order 6 . In fact, $G=\left\{I_{2}, A_{1}, A_{2}, A_{3}, A_{4}, A_{5}\right\}$, where $A_{3}=\bar{\lambda} e_{1} \otimes e_{1}+\lambda e_{2} \otimes e_{2}, A_{4}=\bar{\lambda} e_{1} \otimes e_{2}+\lambda e_{2} \otimes e_{1}$ and $A_{5}=\lambda e_{1} \otimes e_{2}+\bar{\lambda} e_{2} \otimes e_{1}$.

Now we identify $\mathcal{H}=\mathbb{C}^{6}=\mathbb{C}^{2} \oplus \mathbb{C}^{2} \oplus \mathbb{C} \oplus \mathbb{C}$. For a matrix $A$, we denote $A^{(2)}=A \oplus$ A. Put $U_{1}=A_{1}^{(2)} \oplus 1 \oplus 1, U_{2}=A_{2}^{(2)} \oplus 1 \oplus(-1), U_{3}=A_{3}^{(2)} \oplus 1 \oplus 1, U_{4}=A_{4}^{(2)} \oplus 1 \oplus(-1)$ and $U_{5}=A_{5}^{(2)} \oplus 1 \oplus(-1)$ respectively. Then $\mathcal{U}=\left\{I_{6}, U_{1}, U_{2}, U_{3}, U_{4}, U_{5}\right\}$ is a nonabelian unitary group of order 6 .

Let $\xi=\frac{1}{\sqrt{6}}(-1,1,1,1,1,1) \in \mathbb{C}^{6}$; then it is easy to show that $\xi \in \mathcal{W}(\mathcal{U})$. As in Section 2 , we let $\mathcal{M}=W^{*}(\mathcal{U})$. The following result is evident.

Proposition 3. $\mathcal{M}=M_{2}^{(2)} \oplus \mathbb{C} \oplus \mathbb{C}$.

It now follows from Proposition 3 that $E=I_{2}^{(2)} \oplus 0 \oplus 0$ is a minimal central projection of $\mathcal{M}$ such that $E \mathcal{M}$ is a non-commutative factor, while $\left(I_{6}-E\right) \mathcal{M}$ is abelian.

Proposition 4. Let $\mathcal{M}$ and $\mathcal{U}$ be as above. Then $\mathfrak{M}_{\mathcal{U}}$ cannot generate $\mathcal{B}(\mathcal{H})$.

Proof. We know $\mathbb{C}^{6}$ is unitarily equivalent to $\mathbb{L}^{2}(\mathcal{M})$. Then without loss of generality, we may assume that $\mathcal{M}=L(\mathcal{M})$ acts on $\mathbb{L}^{2}(\mathcal{M})$. Let $\Phi \in \mathfrak{M}_{\mathcal{U}}$ and $E$ be as above.

As in the proof of Theorem 1, we may assume that $\Phi$ is a Jordan homomorphism on $\mathcal{M}$ such that $\Phi(I)=I$. Since $E \in \mathcal{M}^{\prime}$, it is sufficient to show that $\Phi L_{E}=L_{E} \Phi$. From the proof of Theorem 1 again, there are two central projections $F$ and $P$ of $\mathcal{M}$ such that $\Phi: F \mathcal{M} \rightarrow P \mathcal{M}$ is a $*$-isomorphism, while $\Phi:(I-F) \mathcal{M} \rightarrow(I-P) \mathcal{M}$ is a $*$-anti-isomorphism. If $F \geq E$, we must have $P \geq E$. Then $\Phi(E)=E$ since $E F \mathcal{M}=E P \mathcal{M}$ is the common non-commutative factor part of $F \mathcal{M}$ and $P \mathcal{M}$. Thus for any $X \in \mathcal{M}, L_{E} \Phi(X)=E \Phi(X)=\Phi(E) \Phi(X)=\Phi(E X)=\Phi\left(L_{E} X\right)=$ $\Phi L_{E}(X)$, which implies that $L_{E} \Phi=\Phi L_{E}$. If $I-F \geq E$, we similarly have the same result. The proof is complete.

Proposition 4 answered the second problem negatively.

\section{ACKNOWLEDGMENT}

The authors would like to thank the referees for their comments and suggestions.

\section{REFERENCES}

[1] X. Dai and D. Larson, Wandering vectors for unitary systems and orthogonal wavelets, Memoirs Amer. Math. Soc., 134(1998), No.640. MR1432142 (98m:47067)

[2] D. Han, Wandering vectors for irrational rotation unitary systems, Trans. Amer. Math. Soc., 350(1998), 309-320. MR 1451604 (98k:47089)

[3] D. Han and D. Larson, Wandering vector multipliers for unitary groups, Trans. Amer. Math. Soc., 353(2001), 3347-3370. MR,1828609 (2002c:46116)

[4] D. Han and D. Larson, Frames, Bases and Group Representations, Memoirs Amer. Math. Soc., 147(1998), No.697. MF1686653 (2001a:47013)

[5] T. W. Hungerford, Algebra, Springer-Verlag, New York Inc., 1974. MR0600654 (82a:00006)

[6] R. V. Kadison, Isometries of operator algebras, Ann. of Math., 54(1951), 325-338. MR0043392 $(13: 256 \mathrm{a})$ 
[7] R. V. Kadison and J. R. Ringorse, Fundamentals of the theory of operator algebras, Vol. II, Academic Press, Orlando, 1986. MR0859186 (88d:46106)

[8] I. E. Segal, A non-commutative extension of abstract integration, Ann. of Math.,57(1953), 401-457. MR0054864(14:991f)

College of Mathematics and Information Science, Shaanxi Normal University, Xian, 710062, People's Republic of China

E-mail address: gxji@snnu.edu.cn

Department of Mathematics, Faculty of Science, Nitgata University, Nitgata, 9502181, JAPAN

E-mail address: saito@math.sc.niigata-u.ac.jp 